\title{
Evaluación y eficacia del branded content: un estudio empírico
}

\author{
Cristina del Pino | cpino@hum.uc3m.es \\ UNIVERSIDAD CARLOS III DE MADRID \\ Ricardo Reinares Lara | rreinares@.usj.es \\ UNIVERSIDAD DE SAN JORGE, ZARAGOZA
}

Resumen: El presente trabajo ofrece los resultados de una investigación centrada en el binomio compuesto por el branded content -formato publicitario consistente en la creación de contenidos por parte de la marca-, y el que según Infoadex es el primer anunciante en términos de inversión publicitaria en España, el grupo El Corte Inglés. La muestra la conforman un conjunto de 361 individuos $y$ el medio en el que se programa el formato de branded content a analizar es Internet. El objetivo es esclarecer la eficacia y utilidad de éste formato, uno de los más significativos dentro del panorama de iniciativas publicitarias que van más allá de la publicidad convencional, y analizar la rentabilidad y pertinencia de este espacio para el anunciante.

Palbras clave: Branded Content, internet, usuario, investigación.

Abstract: This paper focuses on a piece of research between branded content -the advertising format that refers to the creation of content by the brand-, and according to Infoadex, the leading or number one advertiser in terms of advertising investment in Spain, the El Corte Inglés group. The sample is a group of 361 individuals and the format or medium of communication of branded content to be analysed is the Internet. The aim is to gain a better understanding of the efficiency and use/usefulness of this format, one of the most significant within the panorama of advertising initiatives that go beyond conventional advertising, as well as to analyse the profitability and relevance of this form of branded content for the advertiser.

Key Words: Branded Content, internet, user, research 


\section{Perfil del nuevo usuario y consumo audiovisual en Internet: contextualizando el branded content}

La actual situación en materia de comunicación en general y publicitaria de forma concreta, supone una nueva manera de entender el mensaje. Esto significa que en el actual escenario para la comunicación, todos los agentes asumen un nuevo marco de actuación en el que las bases de su funcionamiento han cambiado de forma radical; desde el planteamiento hasta la ejecución, las reglas del juego se escriben al mismo tiempo que se interiorizan y digieren en el contexto del lenguaje digital. La saturación de todos los espacios posibles con publicidad desemboca en una rebelión de la atención, que si bien no es nueva, sí resulta mucho más acusada en los últimos tiempos. Las marcas reinventan su leguaje, tratan de adaptar su discurso a los nuevos medios y los nuevos públicos y sólo las que consiguen hablar en términos de conexión, afinidad y conversación -traducido todo ello a experiencias e impactos-, se encuentran en sintonía para aprender e interiorizar las raíces profundas de este cambio; estamos de lleno en una nueva era en la historia, en la economía, en la cultura, en la sociedad y en general en el individuo, ahora ciudadano y usuario de una realidad 2.0. Ya no se trata de una época de cambios sino de un cambio de época, en donde la Red resulta ser la piedra angular.

Internet se ha convertido en un medio que unifica experiencias tanto de consumo como de ocio, y como tal, ha entrado en una fase de desarrollo y de madurez en que asemeja su naturaleza al automóvil o el ferrocarril en lo que a sus pautas de implantación se refiere. Si atendemos a estimaciones de Price Waterhouse Coopers de 2011, se espera que el crecimiento para el mercado de acceso a Internet hasta 2013 sea de una tasa anual del $9,2 \%{ }^{1}$. Menor Sendrá (2011:137) profundiza en estos datos calculando cómo en los próximos cinco años la tasa de interés compuesto por el conjunto de los servicios digitales será de un $12,1 \%$, frente al $2,6 \%$ de los no digitales.

Calvi y Santini (2011:103) ahondan en el concepto de "la cultura de la red", imprescindible para entender la estructura de la nueva lógica social en general y el consumo de Internet y lo audiovisual en la red en particular, en donde, en sus inicios, nuevas tecnologías de información fueron desarrolladas en el contexto de optimismo tecnológico que desprendían las teorías de MacLuhan (Castells 2001: 47). Aibar (2008), por su parte, analiza a su vez qué tipo de factores sociales o culturales han configurado, no sólo el uso, sino el diseño mismo de Internet, en su evolución desde que naciera en los años cincuenta del pasado siglo; a pesar del tiempo transcurrido, el cambio de modelo experimentado en los últimos dos lustros supera al vivido en los últimos cuarenta años.

El consumo de lo audiovisual en Internet es uno de los pilares de esta investigación, pensando en lo digital como punto de partida. El nuevo consumidor no conoce las fronteras entre el on y el off, y ésta situación ha se ser asumida por parte de las marcas que

${ }^{1}$ Según misma fuente, el crecimiento se espera sea mayor en Latinoamérica, pudiendo superar el $14 \%$. 
pretendan sobrevivir en éste nuevo universo. Según un estudio de Arena Media de abril de $2011^{2}$, conseguir la misma cobertura para una marca cuesta hoy un $23 \%$ más que hace unos años. Otro reciente estudio internacional de Havas Media afirma que solo el 30\% de las marcas supondría de verdad una gran pérdida para los consumidores en el caso de que desaparecieran, y solo el $17 \%$ de las marcas tiene un impacto positivo en el bienestar y calidad de vida del consumidor español ${ }^{3}$. Este mismo estudio, establece una clasificación de las marcas según una escalera de tres peldaños; en el primer escalón, se encuentran las funcionales, en las que lo relevante son los beneficios del producto y el precio; en el segundo escalón nos encontramos con las aspiracionales, con sus significados intangibles y capacidad de diferenciación, y en último lugar, se encuentran las consideradas como meaningful brands, con valores que se perciben como diferenciadores. Todas ellas están teniendo que trabajar duro para adaptarse a esta nueva realidad en donde el nuevo usuario digital ha experimentado una consustancial evolución hasta llegar al estado always on que podemos considerar es, en la actualidad, una de las propiedades más sobresalientes del consumo de lo audiovisual en red. El Ilamado egocastin -definido por Rosen más de un lustro atrás- y la televisión personalizada en el proclamado anyone, anywhere, anytime (Monzoncillo y Sendrá, 2010), constituyen esta nueva realidad en la que Internet implica un cambio cualitativo y nuevas formas de relación social, que de alguna forma, acaban con las relaciones de proximidad. Sobre esta nueva lógica de organización social, las cadenas de televisión encuentran nuevas oportunidades en donde el ocio ocupa un destacado lugar. Son los nuevos eslabones de una nueva cadena y de esta próxima generación del medio rey de comunicación de masas que hasta ahora ha sido la televisión. La integración de ambos actores supone reajustes, nuevos planteamientos así como nuevos modos de distribución. Es por ello, pues, que en este sentido, el negocio de la programación y de la difusión se encuentra en plena efervescencia.

En este nuevo escenario por tanto nos encontramos con que la red vehicula el actual modo de relación que se establece entre el usuario digital y la televisión como medio. Así las cosas, atrás queda la época de audiencias masivas, cautivas y millonarias, donde el televidente se caracterizó por la pasividad frente a unos contenidos regidos por unas normas que hoy difícilmente funcionarían. Asistimos, pues, a un momento en el que cambios sociales se suman a los tecnológicos, nuevos procesos de implantación se aceleran, y medios digitales y soportes han transformado la naturaleza de la audiencia de la genuina televisión generalista. Nuevos formatos surgen al albur de los nuevos medios y en medio de esta situación, la comunicación publicitaria vive uno de sus momentos más convulsos no solo por la caída de la inversión consecuencia de la actual crisis económica, sino por la necesidad de hacer llegar su mensaje a un público cada vez más fragmentado entre los distintos medios y soportes, en donde la tecnología y el lenguaje de redes están cambiado profundamente el lenguaje de la televisión. En los inicios del siglo XXI, ya se hablaba de que la televisión se había

\footnotetext{
2 "Habrá que invertir un 23\% más de GRPs para conseguir la misma cobertura que antes en TV", en: http://www.marketingdirecto.com/actualidad/publicidad/cristina-galan-arena-media-habria-que-invertirun-23-mas-de-grps-para-conseguir-la-misma-cobertura-que-antes-en-tv/

3 "Mainful Brands for a Sustainable Future", en Revista Anuncios, no 1398, 12/12/11
} 
"internetizado" (Pérez de Silva, 2000), y que en la primera década acabaría irrumpiendo la "tercera revolución industrial", la de la convergencia de la Red y de la televisión tradicional. Años más tarde, se han confirmado dichas sospechas al asentarse las claves de un presente multimedia e hipermedia globalizado. En la realidad actual, los profesionales del sector han comenzado a asimilar progresivamente esta nueva filosofía interconectada tras apostar por el lenguaje online y después de ver que el usuario comenzaba a concebir la televisión fuera del salón familiar: ahora para acceder a sus series y programas, recurre al streaming, al método de descarga o al visionado multipantalla, entre otros.

En este sentido, la programación de las cadenas televisivas ha perdido el 'efecto-llamada' tradicional, debido a que los espectadores han comenzado a moldear una parrilla a su gusto. En la década de los noventa del pasado siglo, teóricos como Nicholas Negroponte (1995) ya preconizaban esta sustitución paulatina del prime time televisivo por el my time, aludiendo a las nuevas técnicas para crear una oferta propia y personalizada, gracias a una televisión a la carta accesible a cualquier hora (time-shifting) y desde cualquier lugar (place-shifting).

La transformación experiementada en relación a la televisión y su consumo y el nuevo usuario, es idéntica a la que encontramos en el binomio usuario y nueva comunicación publicitaria. Esta última ha tenido que transformar su apariencia y revestir la imagen de los anunciantes de formatos novedosos y otros que obedecen a una nueva versión de algunos que, habiéndose incorporado al mercado publicitario décadas atrás, han cambiado su esencia para conseguir su supervivencia. Uno de éstos formatos es el branded content, eje de la investigación que presentamos, y que puede ser definido como la creación de contenidos por parte de la marca orientados a mantener conversaciones con el nuevo usuario. Martín (2011) apunta a la importancia de contar una historia con fundamento, basándose en que "puede que no seamos capaces de obligar a los consumidores a ver anuncios, pero sí se puede crear algo que sea tan convincente que los consumidores sientan la necesidad de verlo". En la misma línea, Regueira (2011:218) incide en cómo en la base del branded content se encuentra la máxima "de ofrecer experiencia, no anuncios", es decir, conseguir que el usuario participe, experimente y así, aprenda a través de la involucración. Para este autor, el branded content consiste en un proceso por el que la marca elabora un mensaje, lo coloca en los medios comprando espacios publicitarios -y en los medios compite por la atención del espectador/usuario- dando lugar al paso de una estrategia push - según la cual se impone lo que el anunciante quiere contar- a otra pull-donde es el usuario el que acude al mensaje de forma voluntaria. Reinares Lara (2012) incide en lo importante que resulta diferenciar el branded content de otros formatos publicitarios con los que comparte algunas semejanzas en relación a su naturaleza, pero resultan ser de todo punto prácitcas publicitarias distintas, como da buena prueba de ello el branded content vs el brand placement. En el primer caso, estamos hablando de la creación de un espacio en el que de forma íntegra la marca es la artífice de la idea, dando lugar a programas como el que sirve como objeto de estudio de ésta investigación, el branded content "Tendencias". Independientemente del medio en el que sea emitido, el programa es el resultado de la producción del anunciante, y estará 
presente a lo largo del mismo, recordandolo de distinta manera al usuario/espectador. Por su parte, por brand placement se entiende la presencia puntual e integrada de una marca en el contexto espacial o narrativo del género de ficción, especialmente cinematográfico y televisivo (del Pino y Olivares, 2006: 55). También consideramos importante establecer las diferencias entre el branded content y otros formatos con los que se puede llegar a equívoco en cuanto a su naturaleza conceptual, como por ejemplo el patrocinio o las telepromociones. Se trata de "nomenclaturas diferentes en sus políticas comerciales que definen tipologías idénticas y términos similiares que aluden a tipologías distintas" (Reinares Lara, 2003:193). El patrocinio supone una relación puntual entre la marca y el espacio, de tal forma que mediante las caretas de entrada y salida correspondientes, se comunicará al espectador que una marca "ofrece" un determinado espacio en televisión con el que existe una consonancia de valores que justifica dicha relación. Las telepromociones, por su parte, suponen la recreación publicitaria de las tramas de las series o el aprovechamiento de la notoriedad de un presentador para realizar una comunicación comercial integrada en el contenido. Así, durante un breve periodo de tiempo que no suele superar el minuto, personajes de series o rostros conocidos de una cadena prestan su imagen para interpretar un pequeño guion en el que se suelen incorporar algunos elementos de una serie o programa reconocidos por la audiencia. En ambos casos, los anunciantes no participan de la producción del espacio, cosa que sí sucede en el branded content, desarrollado siempre a partir de las necesidades de comunicación de una marca, con un objetivo de conseguir audiencia. Por tanto, contando con la imprescindible vinculación de marcas y cadenas.

En el caso del branded content, es importante señalar además que se trata del formato publicitario no convencional en televisión en el que de manera más evidente se puede inferir la necesidad del anunciante por la creación de un vínculo emocional o engagement con el usuario/espectador/consumidor. El objetivo último del branded content es la transmisión de valores en un doble sentido: de la marca al contenido y del contenido a la marca, siempre de una manera respetuosa con el objetivo comercial de una cadena, que es conseguir audiencia e ingresos publicitarios. El vínculo entre contenido, marca, usuario, emoción y medio, independientemente de la plataforma en la que se emita el espacio del branded content, es fundamental, y para ello, no vale con la creación de cualquier contenido, sino de uno que se adapte a esta especie de revolución en donde el espectador multitarea exige y demanda una realidad en donde la intencionalidad publicitaria, al menos la que se muestra de forma directa y flagrante, debe quedar diluida.

En definitiva: en el caso del branded content, el espacio creado se integra dentro de la oferta que el medio ofrece porque existe un plus -de naturaleza variada, como entretenimiento e información- que motiva al usuario a consumir ese espacio. Comprobar la eficacia del branded content en un espacio concreto constituye la esencia de la investigación que presentamos a continuación.

\section{Origen, planteamiento y justificación de la investigación}


La presente investigación tiene como objetivo testar el éxito de una propuesta concreta de branded content, un programa denominado "Tendencias" nacido en la primavera de 2012 y emitido en la plataforma de televisión online Mitele.es. Se trata de un nuevo ejemplo de branded content en el que en este caso es la propia marca de El Corte Inglés la que está detrás del contenido. Con este estudio empírico se pretende evaluar los diferentes aspectos del programa, así como la percepción del usuario sobre la presencia del anunciante, El Corte Inglés, en dicho contenido, interesando de forma especial la transmisión de valores entre contenido y marca. El estudio de este anunciante como artífice de la creación de este espacio de branded content , "Tendencias", se justifica porque el grupo El Corte Inglés es, de acuerdo a Infoadex y junto con Procter and Gamble, el primer grupo de anunciantes en el ránking de marcas en 2011, con una inversión de 171,3 millones de euros ${ }^{4}$. A pesar de que esta cifra supone un decrecimiento del $1.7 \%$ con respecto al año anterior, el peso de éste grupo empresarial en términos de inversión justifica que sea objeto de nuestra investigación.

Mediaset España emitió a través de la citada plataforma de televisión online Mitele.es la primera temporada del branded content "Tendencias" con el propósito de ofrecer al espectador información útil contando para ello con profesionales especializados en diversos temas, trucos, consejos, sugerencias y novedades sobre moda. Cada entrega del programa de entre 11 y 12 minutos de duración- lo forman seis microespacios entre los que se incluyen gourmet, shopping o tecnología. Como cierre, un personaje famoso propone tres caprichos para la temporada. La marca está presente tanto al principio como al final de cada entrega con un spot convencional corporativo.

Otros ejemplos de branded content dentro del mismo grupo empresarial los encontramos en Divinity, un canal también perteneciente al grupo Mediaset España que nació como una web sobre celebrities, tendencias y crónica social, y que encuentra en el branded content una inmensa puerta a la colaboración entre medios y marcas, de la cual las dos partes salen claramente beneficiadas. Para la marca supone el estar presente en un espacio de manera menos intrusiva que en la publicidad convencional, y para el medio, permite la creación de programas propios bajo una formula en la que el anunciante financia de forma total o parcial el programa. Así, el programa "Tricotosas", producido por Norit y dirigido a un target muy específico, mujeres entre 16 a 44 años, ofrece como resultado unos picos de audiencia para la cadena del $6 \%$ y de un refuerzo de la presencia del anunciante en la cadena de manera no intrusiva ni agresiva. Otro ejemplo lo podemos ver en el programa "Mis primeras veces" en donde el artífice es la marca Danone y los destinatarios son los padres jóvenes, que protagonizan junto a sus hijos de corta edad espacios de ocho minutos en el que son

\footnotetext{
${ }^{4}$ En 2011, según los datos que ha registrado el Estudio de la Inversión Publicitaria en España 2011 de InfoAdex, hay cuatro sectores que ha dominado la inversión controlada en los medios convencionales. Éstos son automoción, distribución, belleza y finanzas, cuya inversión en conjunto está por encima del $40 \%$ de la tarta publicitaria. Por grupos, El Corte Inglés es el primer grupo anunciante en el ranking de marcas, con una inversión de 171,3 millones de euros aunque ha decrecido un 1,7\%. Le sigue Procter \& Gamble, con una inversión de 123,8 millones y una caída del 6,3\%. En: http://www.marketingdirecto.com/actualidad/publicidad/infoadex-el-corte-ingles-y-pg-los-principalesanunciantes-en-espana-en-2011/
} 
asesoradas por la coach de educación infantil Rocío Ramos Paul, mas conocida como Supernanny. Las familias seleccionadas para el espacio fueron elegidas a través de la página de "Club de Padres" que la propia marca tiene en Facebook, con lo que el circulo de interacción se completa en este caso con el plus de repercusión añadida que supone que esta iniciativa de la red social cuente en Octubre de 2012 con 44.000 seguidores.

\subsection{Metodología de la investigación}

\subsubsection{Muestra}

La investigación se ha llevado a cabo a través de una consulta ad hoc mediante cuestionario on line a espectadores/usuarios del programa, a través de un link incrustado en los propios sitios donde se emite el programa, en Mitele.es, solicitando su opinión sobre los contenidos vistos. La muestra final alcanzada es de 361 espectadores, a los que hay que sumar una muestra control externa de 200 individuos no expuestos a "Tendencias", a los que de manera paralela se aplicó una entrevista online, valorando los mismos atributos de imagen del anunciante propuestos a los espectadores de "Tendencias". Con esta información y por comparación, se puede cuantificar la aportación neta del programa a la imagen de marca del anunciante, El Corte Inglés.

\subsubsection{Perfil del espectador del programa}

La distribución del espectador del espacio "Tendencias" se ha llevado a cabo considerando la distribución por sexo, por actividad y por edad, haciendo una distinción entre el programa en sí y el portal en que éste se emite, Mitele.es. De esta forma los resultados nos llevan a un porcentaje algo superior de mujeres -un $81 \%$ - frente a hombres - 19\%-como espectadores de "Tendencias", siendo la distribución por sexo en general en el portal Mitele.es del 64\% de mujeres frente al $36 \%$ de hombres. En cuanto a la ocupación o actividad de los componentes de la muestra con respecto al programa, destaca un alto porcentaje de usuarios activos -el $53 \%$ - frente a los inactivos $-15 \%$ - y los estudiantes, que suponen una cifra representativa a tenor del 32\% que constituyen. En cuanto a la distribución en general -según la actividaden el citado portal, los usuarios activos nuevamente representan la cifra más elevada alcanzando más del $60 \%$ de la muestra, mientras que los inactivos y los estudiantes alcanzan el $40 \%$ del resto (12 y $28 \%$ respectivamente). Por último, el cuanto a la distribución por edad, el mayor porcentaje de individuos tienen una franja de edad de entre 21 y 45 años, perteneciendo el 68\% de ellos al programa "Tendencias" propiamente dicho, y el $44 \%$ al portal Mitele.es. La representación gráfica de la radiografía del perfil del espectador de "Tendencias" según su distribución por sexo, actividad se puede apreciar en el gráfico 1. 


\section{Resultados de la investigación}

Los resultados de la investigación arrojan luz sobre un amplio espectro de campos de los que se ha obtenido información variada. En primer lugar, hemos considerado una serie de indicadores que ofrecen información cuantitativa sobre la eficacia del programa en sí, un formato on line testado sobre un grupo de usuarios que de considerar como positiva esta acción de branded content, podría suponer que el anunciante se planteara el paso de la emisión de "Tendencias" del mundo online que supone esta plataforma de Internet, al mundo offline que representa la televisión. Por tanto, resultaba de todo punto interesante en primer lugar medir la notoriedad y la consideración del anunciante con respecto al programa; a continuación pretendimos averiguar la adecuación de "Tendencias" para la imagen de marca del anunciante, amparándonos para ello en si es o no pertinente la apuesta de un anunciante como el Corte Inglés en un formato como el branded content. En tercer lugar, quisimos averiguar cuales eran los significados asociados al anunciante por su patrocinio/producción del programa, una información cuantitativa y cualitativa para dar luz a cuestiones relacionadas con la percepción de los consultados sobre la asociación de la imagen de marca del anunciante al formato; en cuarto lugar pretendimos conocer cual era la percepción y la valoración del programa en sí, es decir, si "Tendencias" funciona como programa independiente, si esta acción de branded content gusta o no y hasta qué punto. Interesante en éste sentido fue averiguar el desglose por géneros de la muestra. Dentro de éste apartado, también constituía una información valiosa conocer cuales son las secciones del programa que más gustan, con el claro objetivo de potenciar las más valoradas en sucesivas entregas y en el otro extremo, mejorar las peor evaluadas o incluso pensar en su eliminación. Por último, resultaba esclarecedor conocer la información referida a los valores del branded content "Tendencias", una información sobre intangibles rica en matices para el anunciante, que verá en posteriores estadíos de que manera sacar jugo a ese conjunto de atributos sobre los que los usuarios consultados se pronuncian en este apartado. La información obtenida de todos los puntos tratados se desglosa a continuación.

\subsection{Indicadores de eficacia: la notoriedad y la consideración del anunciante con respecto al} programa

\subsubsection{Indicador de Notoriedad del anunciante}

El espacio de branded content que estudiamos presenta el patrocinio-producción del anunciante que lo produce, El Corte Inglés. Tomando el indicador notoriedad como base, resulta especialmente significativo que tres de cada diez espectadores cita espontáneamente el nombre del anunciante como patrocinador de Tendencias. De forma sugerida, el reconocimiento asciende a seis de cada diez espectadores. Esta cifra puede ser traducida como muy positiva para la marca, teniendo en cuenta el nivel de saturación publicitaria a la que está sometido el espectador medio, el cual es objeto de un contínuo bombardeo 
publicitario que le hace desarrollar resistencia y oposición a las marcas y a todo mensaje, con apariencia publicitaria o no, que provenga de ellas. El recuerdo de marca, de manera natural o siendo provocado, indica que de alguna forma este anunciante ha superado el ostracismo al que el usuario somete a las firmas comerciales como resultado de la citada saturación. La representación gráfica de los resultados de la notoriedad en relación al recuerdo son los que se pueden leer en el gráfico 2 .

Otro dato significativo en el estudio de la notoriedad del anunciante, es el derivado del desglose de la muestra por sexo, edad y número de programas vistos. Así, el estudio determina que la notoriedad de El Corte Inglés en "Tendencias" es considerablemente superior entre los espectadores masculinos y creciente a mayor edad. También, como cabía esperar, un mayor número de programas visto mejora en gran medida las cifras de identificación de la marca

\subsubsection{Indicador de Consideración del anunciante}

Entre los espectadores de "Tendencias", la consideración de El Corte Inglés como lugar favorito de compra es de un $43 \%$ superior a la manifestada por los no espectadores. En una escala de cero (nada) a diez (mucho), se ha formulado a los espectadores la pregunta de "hasta que punto crees que tienes en cuenta a esta firma comercial como un lugar favorito de compra". Entre las respuestas, el rechazo -considerando como tal una puntuación de entre 0 y $4-$ ha sido del $25 \%$ entre los espectadores y del $40 \%$ entre los no espectadores. Siguiendo con la escala, y tomando en este indicador la puntuación entre 5 y 6 de esta consideración, el porcentaje entre los espectadores y los no espectadores es prácticamente igual: un $18 \%$ de los espectadores se posicionan como indiferentes a la pregunta y éste porcentaje sube dos puntos $-21 \%$ - entre los no espectadores. En cuanto a la aceptación, que se corresponde con las cifras de 7 a 10 en nuestra escala, el 56\% de los espectadores conforman la aceptación frente a la pregunta formulada mientras que esta cifra baja al 39\% entre los no espectadores. Considerando la variable sociodemográfica sexo y edad en éste indicador, resulta significativo como el incremento en consideración de El Corte Inglés es positivo en todos los grupos de espectadores pero muy llamativo entre las mujeres, y sobre todo, las más jóvenes. Las diferencias son también significativas aún cuando se trata de espectadores que sólo han visionado de 1 a 4 programas, llamados "espectadores light" - y máximas cuando el visionado supera los cinco programas, los considerados como "espectadores heavies".

\subsection{Adecuación de Tendencias para la imagen de marca del anunciante}

La aludida saturación publicitaria es el principal hándicap con que cuenta hoy un anunciante que pretenda sobresalir y llegar al usuario; para evitarla, la medida adoptada por El Corte Inglés como marca publicitaria al inclinarse por el branded content, presenta un abanico de 
posibilidades que bien explotado, supone una puerta abierta a la creación de contenidos y todo el beneficio que de ésta acción se deriva. Cuanto menos se perciban esos contenidos como el resultado de la producción de una marca comercial, mejores repercusiones habrá para la imagen de marca. En este sentido, la pregunta formulada a la muestra es clara: "¿cómo te parece de adecuado que El Corte Inglés produzca- genere- patrocine programas con contenido de éste tipo? La respuesta fue que dos de cada tres espectadores consideraron como adecuada éste tipo de iniciativa para la imagen del anunciante, mientras que al resto le parece indiferente; solo uno de cada diez espectadores manifiesta una opinión contraria en éste sentido. Observando las características de los espectadores, se aprecia que la aceptación es mayoritaria en todos los grupos, pero superior a la media en los más jóvenes y los llamados "espectadores heavies". No hay un posicionamiento diferente en relación al sexo de los encuestados considerando la pregunta formulada, suponiendo un porcentaje prácticamente idéntico. Gráficamente, éste resultado se traduciría de la forma en que se puede ver en el gráfico 3.

Otro resultado muy indicativo de la situación, son las conclusiones a las que llegan los espectadores cuando explican cómo de adecuado consideran éste formato para una marca como El Corte Inglés; tres conclusiones son las que consideramos cómo relevantes a éste respecto. En primer lugar consideran que es un programa innovador acorde con la imagen de marca de el anunciante; en segundo lugar, consideran que este tipo de iniciativas puede ser un gran escaparate de la variedad de productos disponibles que éste anunciante ofrece al mercado, y por último, los espectadores consideran que se transmite una buena imagen del patrocinador, estando acorde con lo que se espera de una marca posicionada como El Corte Inglés. Sólo el $10 \%$ de los espectadores piensa que el formato es poco o nada apropiado, y las razones que argumentan para ello no están relacionadas en sí mismas con el propio formato en sí, sino en la mayor parte de los casos, con la percepción de que se trata de un contenido publicitario. Entraríamos por tanto en éste sentido en el campo de las percepciones derivadas de la publicidad encubierta y de los formatos que en esta línea, intentan vender un mensaje como no comercial cuando en realidad lo es.

\subsection{Significados asociados a la marca por su patrocinio del programa}

Bajo éste parámetro quisimos averiguar qué era lo que realmente quería transmitir el anunciante con esta acción de branded content. La idea era saber si el objetivo perseguido lo era en términos de marcar tendencias, renovarse o modernizarse, entre otras metas, y los resultados resultaron ser del todo esclarecedores. Entre las respuestas obtenidas, la muestra coincidió en que la imagen de cercanía y variedad es un indicativo de calidad y de empresa que destaca frente a otras del sector por un estilo más vanguardista, que trata de dejar atrás otros valores o perfiles de otra índole más relacionados con la seriedad y lo clásico. Este anunciante pues, al crear espacios de este tipo, se decanta por una apuesta atrevida que al mismo tiempo les acerca al público joven, lo que supone por sí mismo un 
acercamiento a un sector de población al que históricamente esta marca ha querido llegar. En el extremo menos positivo, destacar como en algunos casos, el programa "Tendencias" se percibe como una forma de publicidad más, cuyo objetivo es la venta y el fomento del consumismo. Preguntada la muestra en una escala de cero (nada) a diez (mucho) sobre hasta qué punto creen que patrocinando este programa da muestra de ser una compañía moderna, de calidad, joven, útil o dinámica, los resultados fueron los que se pueden ver en el gráfico 4.

\subsection{Percepción y valoración de "Tendencias" como programa}

En esta parte de la investigación, el objetivo era averiguar si el programa como tal gusta o no a los consultados. En función de los resultados se podría saber si el formato funciona y si es una apuesta segura para el anunciante, tanto por si merece la pena seguir hacia adelante con otros programas similares como por trasladar la apuesta del mundo on line al off line. Resulta mucho más económico para un anunciante testar el programa en Internet, y en función de los resultados de la investigación, llevar a cabo la inversión necesaria para llevarlo al medio televisión, que probar directamente en éste medio que requiere una mayor inversión económica. El resultado fue que la aceptación del programa fue muy alta a juzgar por los datos obtenidos: en total, siete de cada diez consultados afirman que el programa les ha gustado mucho o bastante, mientras que para el resto de los espectadores el programa ni gusta ni disgusta, representado el rechazo una cifra del $9 \%$ de los consultados

Considerando la variable sexo y edad, la aceptación del programa es mayoritaria en todos los grupos, destacando levemente entre las mujeres y el corte de mayor edad. La muestra coincide en afirmar que la mayor fortaleza del programa reside en sus contenidos y en el dinamismo con que éstos son contados, mostrándose las últimas novedades de forma amena, rápida y clara, resaltando lo que puede resultar de más interés. En el otro extremo, destacar como lo que menos gusta del programa resulta ser que se percibe como publicidad y que es elitista, al mostrarse las novedades que se presentan como bienes de consumo con un coste económico alejado de lo que el ciudadano medio puede invertir en ellos. Por otra parte, y dentro de la misma variable, se quiso averiguar cuales son las secciones del programa que más interés despiertan y que más gusta a la muestra, siendo la respuesta mayoritaria la sección moda, seguida de la sección belleza, espectáculos, tecnología, gourmet y shopping.

\subsection{Valores y atributos de Tendencias}

Por último, quisimos saber hasta que punto los usuarios podrían pensar que el programa en sí era un contenedor que transmitiera entre otros los valores de intensidad, emoción, cercanía o confianza. Teniendo en cuenta la amplia oferta existente de anunciantes que buscan la manera de transmitir estos intangibles, resultaba de todo punto esclarecedor 
conocer si la muestra reconocía éstos atributos en éste programa y con un patrocinador como El Corte Inglés. Se trata de valores que pueden estar presentes de forma abstracta en multitud de mensajes dentro de la comunicación publicitaria convencional, pero la combinación de éste programa y ésta marca como creadora de contenidos es un binomio que puede aportar luz sobre la calidad, el dinamismo o la modernidad/actualidad de una apuesta de este tipo. En efecto, este último atributo es reconocido por el $72 \%$ de la muestra, y en el extremo de menor peso en términos porcentuales, reconocemos el valor de la intensidad y la emoción (43\%) a un formato que por todo lo demás, se encuentra en pleno crecimiento y del que consideramos con estas cifras aún debe seguir su proceso de ir puliendo las aristas que ésta investigación arroja. Es importante señalar que no existen diferencias significativas por géneros en relación a éstos atributos, como se puede ver en le gráfico 5 .

\section{Conclusiones generales}

Los resultados de ésta investigación suponen una valiosa información tanto para el anunciante como para el formato en sí. Consideramos que el estudio de la eficacia de una acción de branded content en una plataforma online, por un lado, y la pertinencia o no de una marca posicionada en el mercado décadas atrás como El Corte Inglés como productora de ése espacio, por otro, puede aportar conocimiento del sector a otros anunciantes y al mercado publicitario en general, habida cuenta del problema de la saturación de los espacios publicitarios convencionales y de la fragmentación de las audiencias en éstos, especialmente, en la televisión. Así pues, entre los resultados más significativos y específicos del anunciante, cabe destacar que esta acción de branded content capta a espectadores jóvenes con un peso muy importante de mujeres, mayoritariamente compuesto por activos y estudiantes. Otro dato igualmente interesante a resaltar como conclusión, es que ser espectador de "Tendencias" se traduce en un $43 \%$ de incremento en la consideración del El Corte Inglés como lugar favorito de compra, existiendo así mismo una transmisión de valores entre el formato de branded content y el anunciante muy fuerte: la marca se reviste de fuertes enganches emocionales, como la cercanía y la intensidad, además de ser considerada como original, innovadora y joven. Así mismo, cada una de sus secciones resulta ser muy relevante para los diversos segmentos de los espectadores, complementándose éstos de forma satisfactoriamente equilibrada.

En cuanto al formato en sí, resaltar cómo "Tendencias" supone una acción de branded content fruto del acuerdo del anunciante con la cadena; siguiendo la estela de este anunciante, otros muchos se sumaron antes y después a la creación de contenidos con mayor o menor suerte en términos de rentabilidad del espacio; no todos los casos de branded content han resultado ser de éxito, si bien muchos de ellos han sido objeto de una alta notoriedad. Prueba de ello lo encontramos en el programa "Fix You", de Turespaña, en MTV, que fue concebido como una serie de televisión de cuatro capítulos emitidos en Alemania, Reino Unido, Corea y China, con un potencial turístico muy importante para 
nuestro mercado, pues se trata de un reality show donde los protagonistas tratan de corregir el rasgo de su personalidad menos aceptado por su entorno. Los enclaves elegidos pertenecen a la geografía española donde la diversión, el clima y el carácter español son parte del atractivo turístico. La intención es resaltar puntos fuertes de nuestro país, vender España fuera de nuestras fronteras, marcar tendencia, o despertar el deseo de ser elegido como destino turístico.

La clave es identificar qué es importante para el anunciante pero por encima de él, para los consumidores, con el objetivo de ofrecer un producto de interés, siendo un elemento diferenciador para un target cada vez más inmunizado contra la publicidad y que necesita saber quién le habla, qué quieren, qué sienten y de qué manera. Otro ejemplo significativo lo constituye ING, el primer anunciante que ha apostado en Antena 3 por la creación de contenidos. En un espacio del mismo nombre, llamado "Aprender a desaprender", se lleva a cabo un llamamiento a pensar de forma diferente y a replantearse las reglas establecidas, potenciando de esta manera una forma de actuar distinta. La línea del programa gira en torno a la experiencia de seis conocidos personajes de diferentes campos, que cuentan cómo por diferentes circunstancias tuvieron que plantearse los hábitos hasta el momento adquiridos para dar un nuevo giro y afrontar situaciones de otra forma. Rostros conocidos del mundo del deporte o el diseño contaron en el espacio lo que significó para ellos salirse de lo establecido. El programa contó con avances de programación emitidos en pauta única en el grupo Antena 3, lo que permitió según fuentes de la cadena impactar a más de 30,6 millones de espectadores alcanzando a más del $73 \%$ de la población. Por su parte, el banco Sabadell se decidió por una acción similar de branded content con la creación de una serie de diálogos con personajes públicos relevantes con una cuidada estética en blanco y negro con la que se pretendió hacer un guiño a la televisión desaparecida, la clásica, la primera televisión. La acción centrada en una serie de vídeos alojados en YouTube, resultan ser conversaciones más o menos profundas que llevan a la web del banco, creando diálogo sobre posibles soluciones a problemas. De esta forma, el banco financia un contenido relevante sin intención de venta, en donde se pone de manifiesto la mutación de la inversión en comunicación que interrumpe por otra muy diferente que lejos de interrumpir, vincula.

El branded content, en suma, está siendo tratado de manera profesional por numerosos anunciantes, que han intentado con ahínco resetear la creatividad en su apuesta por la creación de contenidos, obviando planteamientos relacionados con mensajes convencionales o con publicidad trescientos sesenta grados. El objetivo es buscar la esencia, una idea que resuelva un problema, una solución inesperada con resultados y el reto ante el cambio.

Otra de las conclusiones significativas de éste estudio que se puede aplicar de manera genérica es el cómo queda reflejado que la mayoría de los usuarios receptores de éste formato resultan ser los jóvenes, ratificando la teoría de Agnola y Le Champion (2003:126) que más de un lustro atrás dieron cuenta de cómo los mayores usuarios de la nueva forma de ver televisión a través de Internet son los jóvenes, pues sus aspiraciones y los valores de las nuevas tecnologías encuentran evidentes puntos de coincidencia con la interactividad, 
virtualidad, P2P y en general, las llamadas "tecnologías de relación", como el podcasting, blogging o redes sociales (Gallego Pérez, 2009:68). Precisamente, la personalización y la individualización son los artífices de los cambios sociales de la nueva televisión personal, en donde en general puede hablarse de una nueva sociedad en la que asistimos a una reinvención de la televisión a través de la red.

El nuevo usuario consumidor de branded content en particular, y de formatos publicitarios no convencionales en general, está encarnado en un nuevo espectador que ante todo, participa, opina, produce, recomienda o descarga en una nueva manera de entender el medio. En este nuevo panorama se sigue probando la eficacia de nuevos formatos, los anunciantes se lanzan y atreven con formatos poco explotados hasta ahora $y$, entre otros, se hablan de nuevos protocolos en el medio - IPTV (Internet Protocol Television), o, siguiendo a Noam (2008:122) de crear, entre otros, nuevas formas y géneros basados en la creatividad e interactividad, en la producción y en la difusión social de información, como resultado de la acción colaborativa de millones de personas que participan de forma directa o indirecta y que ejercen una influencia unas sobre otras (Calvi y Santini, 2011:108).

Este trazado del nuevo usuario es a su vez el de los nuevos medios, un concepto amplio, que integra y difunde los medios del pasado y enlaza con el denominado por Kennedy (2008:315) como "el lenguaje de lo potencial" y el "altar" de la convergencia (Jenkins, 2008: 15), un nuevo paradigma para entender los cambios en los media, especialmente los directamente relacionados con el origen de lo audiovisual en Internet y la convulsa realidad resultante del binomio formado por el consumo audiovisual y la red de redes. La comunicación publicitaria, por su parte, se integra y asume los cambios acontecidos en este panorama en continua efervescencia.

\section{Referencias bibliográficas}

Agnola, Michel y Le Champion, Rémy (2003): La televisión sur internet. Francia. Editorial Puf.

AIBAR, Eduard (2008): "Las culturas de Internet: la configuración sociotécnica de la red". Revista Iberoamericana de Ciencia, Tecnología y Sociedad. Ciudad autónoma de Buenos Aires.

CALVI, Juan Carlos, y SANTINI, Rose M (2011): "La cultura de Internet" en MONZONCILLO, José María (2010): La televisión etiquetada: nuevas audiencias, nuevos negocios. Madrid, Ed. Ariel y Fundación telefónica.

CASTELls, Manuel (2003): La galaxia de Internet, Barcelona, Planeta. 
Del Pino, Cristina y Olivares, Fernando (2006): Brand Placement: Integración de las marcas en la ficción audiovisual. Evolución, casos, estrategia y tendencias. Barcelona, Gedisa.

Gallego Pérez, Ignacio (2009): "Podcasting: distribución de contenidos y nuevas formas de negocio en la empresa radiofónica española, Tesis Doctoral, Universidad Complutense de Madrid.

Jenkins, Henry (2008): Convergence Culture: where old and Media Collide. New York University Press.

KenNedy, Henry (2008): "News Media's Potential for Personalization and Information" Communication \& Society 11(3).

Menor SendrÁ, "El dinero audiovisual" en MONZONCILlO, José M. (2010): La televisión etiquetada: nuevas audiencias, nuevos negocios. Madrid, Ed. Ariel y Fundación telefónica.

Monzoncillo, José M., y Sendrá, Juan (2010): "La televisión, entre la gratuidad y el pago". Madrid, Revista Telos. Cuadernos de Comunicación e Innovación.

NoAm, Eli (2008): The Economics of User Generated Content and Peer to Peer. The comons as the Enabler of Commerce. New York. Ed. Springer.

PÉREZ DE SILVA, Javier (2002): La televisión ha muerto. Barcelona, Editorial Gedisa.

RegueIRA, Javier (2011): Big Brother is dead. El día que el consumidor hizo callar a las marcas. Madrid, ESIC.

ReINARES Lara, Ricardo (2003): Formatos no convencionales de Publicidad en Televisión, Madrid, ESIC.

Rosen, Cristhine, "The Age of Egocasting", The New Atlantis: the Journal of technology and society. Fall 2004-Winter 2005.

[Recibido: 10 de diciembre de 2012. Aceptado: 14 de octubre de 2013]. 

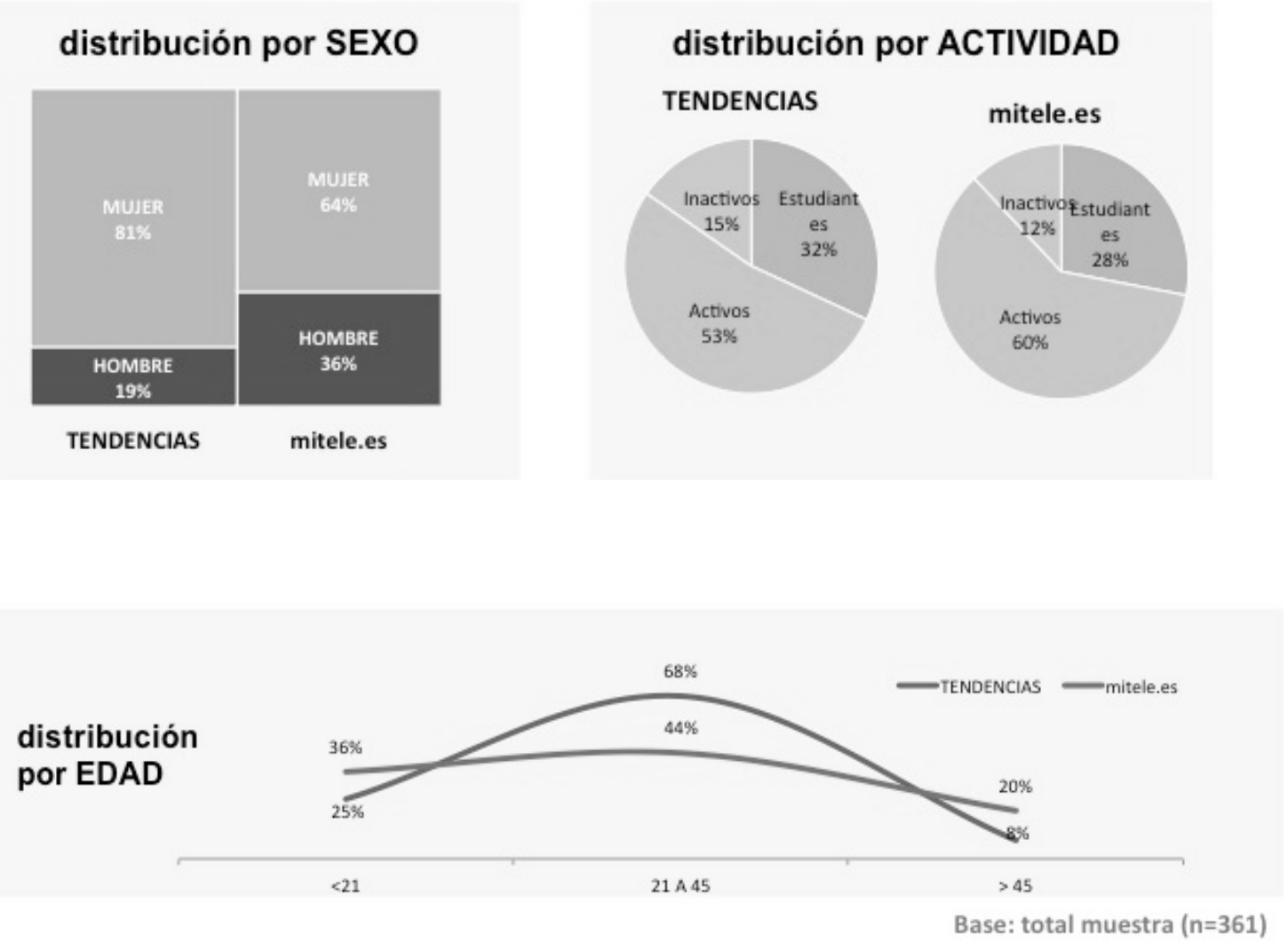

Gráfico 1. Distribución de la muestra por sexo, actividad y edad.

Notoriedad de ECI en TENDENCIAS

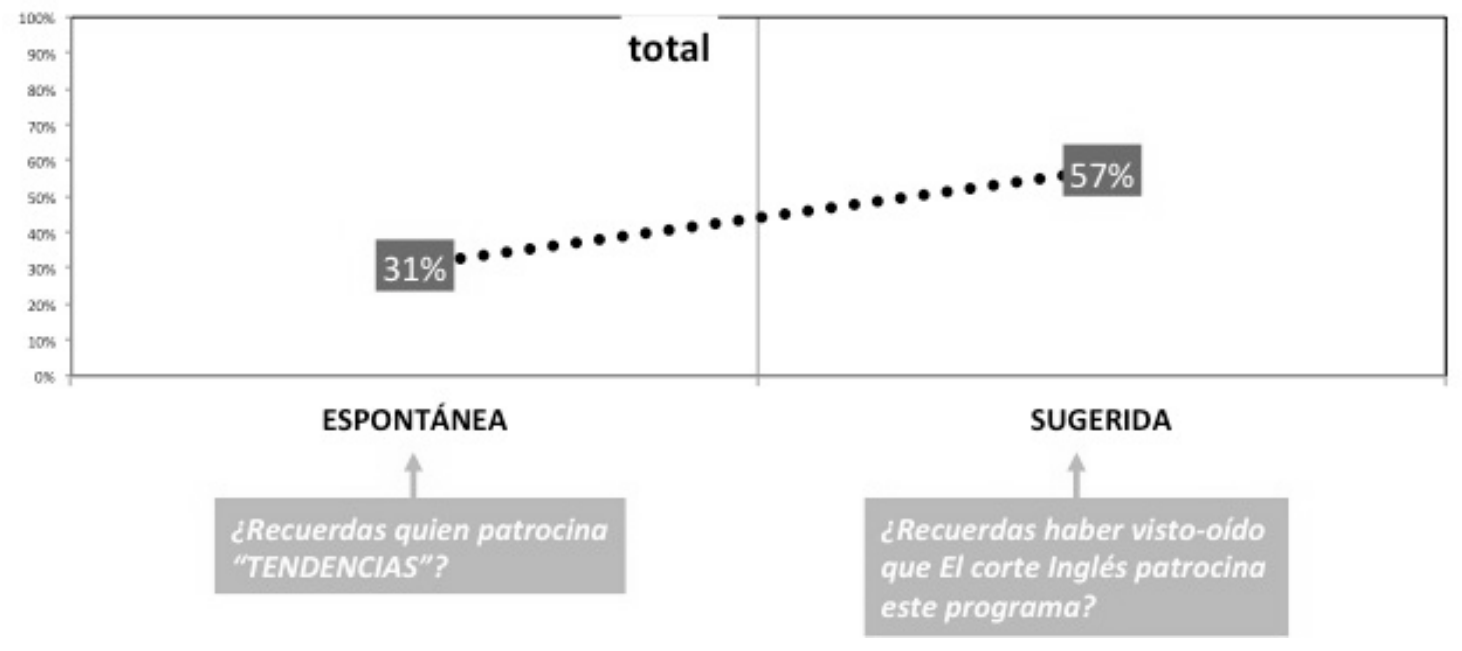

Gráfico 2. Notoriedad del anunciante en Tendencias según recuerdo de marca 


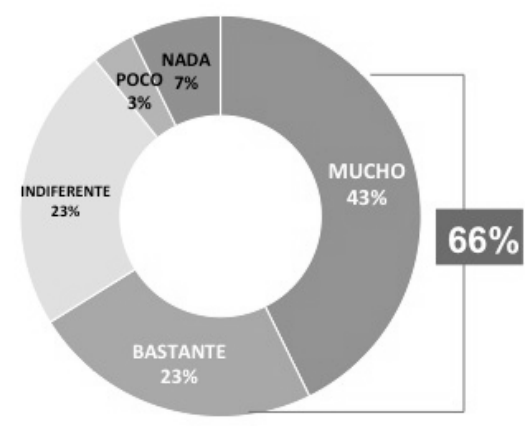

Gráfico 3. Adecuación de Tendencias para la imagen de marca del anunciante

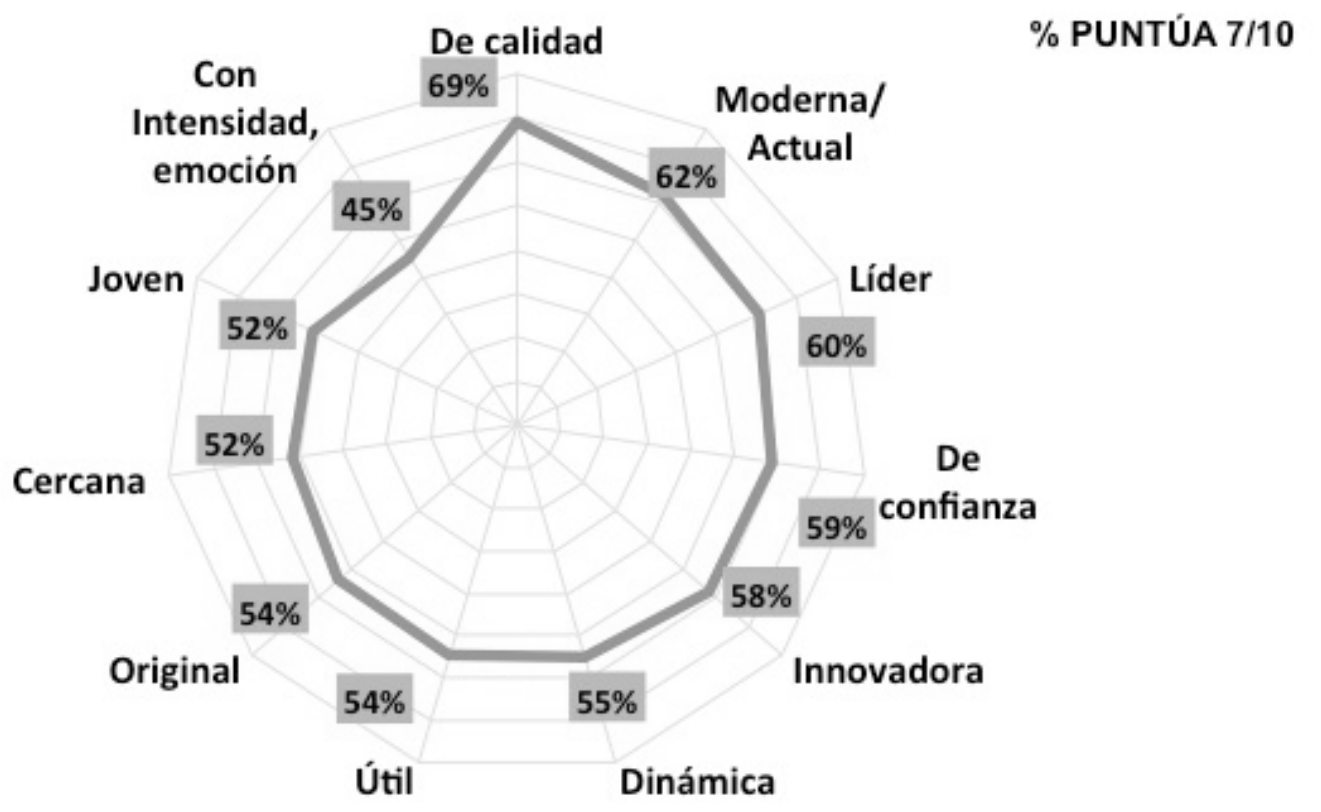


Gráfico 4. Significados asociados al anunciante por su patrocinio del programa

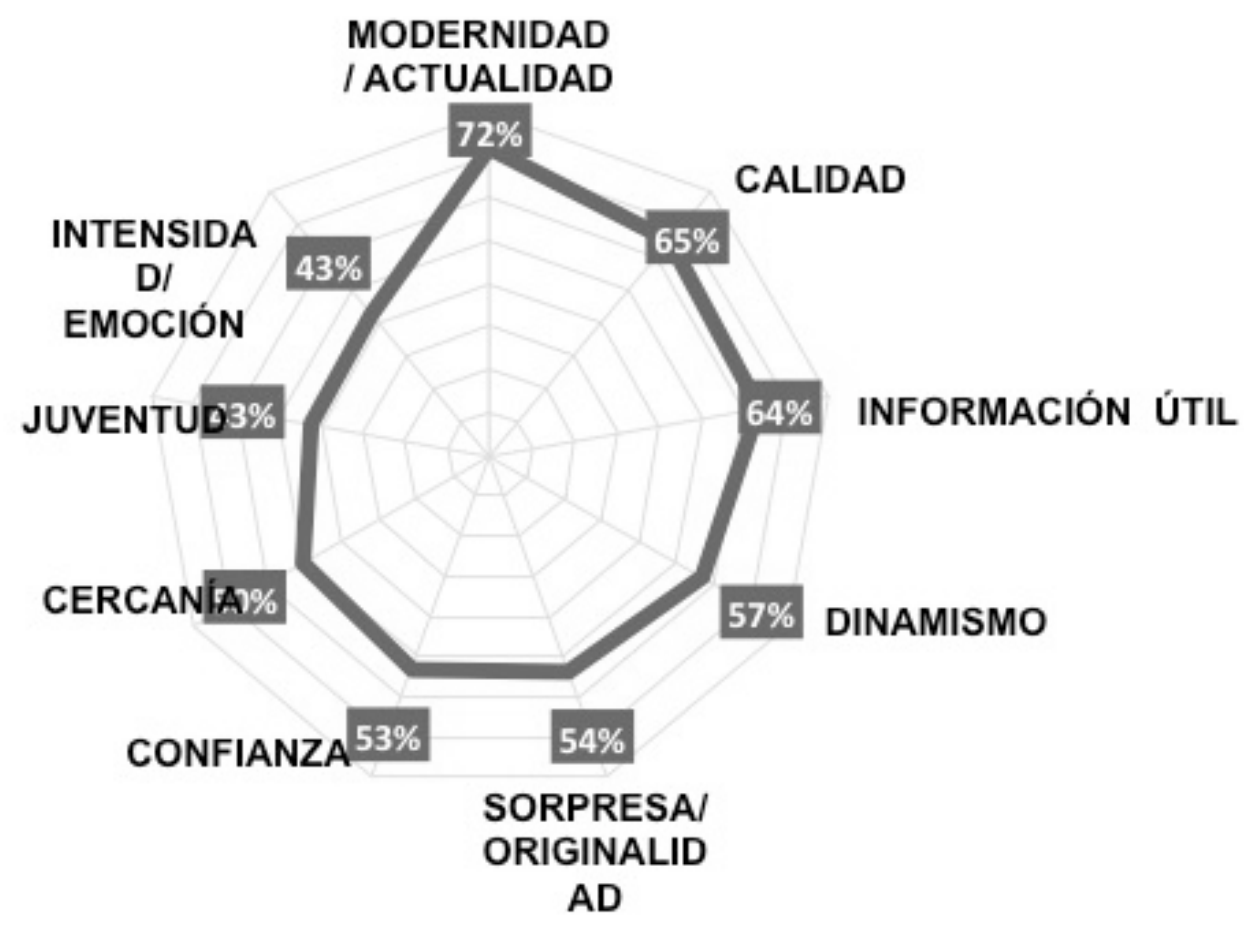

Gráfico 5. Valores y atributos de "Tendencias" 